\title{
Emergências médicas em consultório odontológico
}

\author{
Medical emergencies in dental practice
}

MORETTO, Marcelo Juliano'; MENEZES, Lelis Borges de ${ }^{2}$; BARBIERI, Gabriel ${ }^{2}$; MENEZES, Pedro Rafael ${ }^{3}$

\footnotetext{
1. Doutor em Ciência Odontológica. Professor da Universidade de Rio Verde, Rio Verde, GO, Brasil.

2. Graduado em Odontologia, Faculdade Morgana Potrick, Mineiros, GO, Brasil.

3. Graduando em Medicina, Faculdade Morgana Potrick, Mineiros, GO, Brasil
}

Endereço para correspondência:

Marcelo Juliano Moretto

Universidade de Rio Verde

Curso de Odontologia

Fazenda Fontes do Saber

Caixa Postal 104

75901-970 - Rio Verde - Goiás - Brasil

E-mail: mjmoretto@yahoo.com.br

Recebido: 08.05.2017

Aceito: 30.06.2017

\section{RESUMO}

Este estudo teve como objetivo avaliar o conhecimento dos acadêmicos do último ano do curso de Odontologia da Faculdade Morgana Potrich sobre o conhecimento em emergências médicas em consultório odontológico. Foi realizado um estudo transversal descritivo desenvolvido por meio de dois questionários semiestruturados aplicados a 43 acadêmicos. O primeiro questionário continha questões básicas autoavaliativas e um segundo questionário com questões elaboradas sobre o conhecimento dos alunos sobre os diferentes tipos de emergências e condutas. Como resultados observamos a grande insegurança dos alunos da instituição diante de situações de emergência. Houve grande interesse pela maioria dos graduandos em obter mais informações e treinamento em suporte básico de vida (SBV).

Palavras-chave: Emergências. Consultórios odontológicos. Medicina de emergência.

\begin{abstract}
The aim of this study was to evaluate the knowledge of last year's scholars of the Dental School of Morgana Potrich on the knowledge of medical emergencies in a dental office. A cross-sectional descriptive study carried out through two semistructured questionnaires applied to 43 academics. The first questionnaire contained basic self-assessment questions and a second questionnaire with elaborate questions about students' knowledge of different types of emergencies and behaviors. As a result, observed the great insecurity of the students of the institution in the face of emergencies. There was great interest from most undergraduates in obtaining more information and basic life support training (SBV).
\end{abstract}

Keywords: Emergencies. Dental offices. Emergency medicine. 


\section{INTRODUÇÃO}

O cirurgião-dentista como profissional de saúde tornase responsável pela vida de seu paciente estando suscetível a deparar-se com situações de emergência médica em seu cotidiano, podendo ocorrer antes durante ou depois de procedimentos simples ou invasivos ${ }^{1-3} . \mathrm{O}$ avanço da medicina tem possibilitado cada vez mais que pessoas acometidas sistematicamente pudessem ter uma melhora significativa em sua qualidade de vida, desta forma podemos perceber uma maior diversidade de pacientes acometidos por enfermidades sistêmicas que procuram atendimento odontológico tendo a consciência de que a saúde bucal está diretamente ligada a manutenção da saúde geral $^{3-5}$.

A emergência médica é um evento inusitado ou inesperado que requer uma avaliação rápida devido à alteração súbita da condição sistêmica e fisiológica do indivíduo, podendo levar ao risco de morte6. Aproximadamente $75 \%$ dos casos de urgências e emergências médicas na prática odontológica são desencadeados por estresse e medo ${ }^{7-8}$. Dentre as urgências e emergências médicas de maior ocorrência em consultório odontológico destacam se: síncope, convulsão, reação alérgica, obstrução de vias aéreas, hipoglicemia, emergências cardiovasculares e crise de asma ${ }^{9-14}$.

Lamentavelmente acadêmicos de odontologia e cirurgiões-dentistas esporadicamente sentem segurança para atender a uma emergência médica ${ }^{4,15}$. A defíciência de matérias e disciplinas na área de emergências médicas durante a prática odontológica leva a dificuldade de entendimento entre os profissionais e acadêmicos. Mesmo que grande parte dessas emergências não represente perigo eminente de morte para o paciente, é imprescindível que o cirurgião-dentista esteja apto a lidar com um evento emergencial, pois apenas o preparo poderá contribuir para a redução da morbidade e mortalidade nessa situação ${ }^{16-20}$.

A grande divergência entre os acadêmicos sobre o conhecimento, identificação e tratamento das emergências médicas em consultório odontológico, tornam a avaliação da formação e da conduta dos alunos dos últimos períodos do curso de odontologia da Faculdade Morgana Potrich diante de uma situação de emergência. Os questionários utilizados no estudo foram realizados no intuito de identificar possíveis falhas durante a formação e na necessidade de treinamento contínuo para a formação de profissionais mais conscientes e aptos a atenderem as situações de emergência, com segurança e conhecimento técnico.

\section{MATERIAL E MÉTODOS}

O estudo denominado "Emergências médicas em consultório odontológico" foi registrado e aprovado no comitê de ética em pesquisa sob o no CAAE: 58217316.2.0000.5428.

Foi realizado um estudo transversal descritivo por meio da aplicação de questionário em sala de aula com tempo cronometrado e sem que houvesse comunicação entre os alunos, após a assinatura do termo de consentimento livre e esclarecido.

Para a coleta de dados utilizou-se dois questionários semiestruturados, elaborados pelos pesquisadores, no primeiro questionário foi abordando dados da amostra assim como informações sobre o atendimento de emergências médicas em consultório odontológico e no segundo foram elaboradas questões avaliativas sobre o atendimento emergencial, foi utilizado como parâmetro para identificar se o julgamento dos alunos sobre o conhecimento relatado no primeiro questionário condiz com a realidade. $\mathrm{O}$ segundo questionário só foi entregue após o preenchimento e entrega do primeiro.

A amostra foi composta por 43 alunos devidamente matriculados no último ano do curso Odontologia da Faculdade Morgana Potrich (FAMP), que concordaram com os termos da pesquisa e assinaram o termo de consentimento livre e esclarecido.

\section{RESULTADOS}

Para avaliação do conhecimento dos acadêmicos da FAMP foram elaborados dois questionários sobre emergências médicas em consultório odontológico, foram abordados 43 alunos sendo $77 \%$ do gênero feminino e $23 \%$ do masculino (Figura 1).

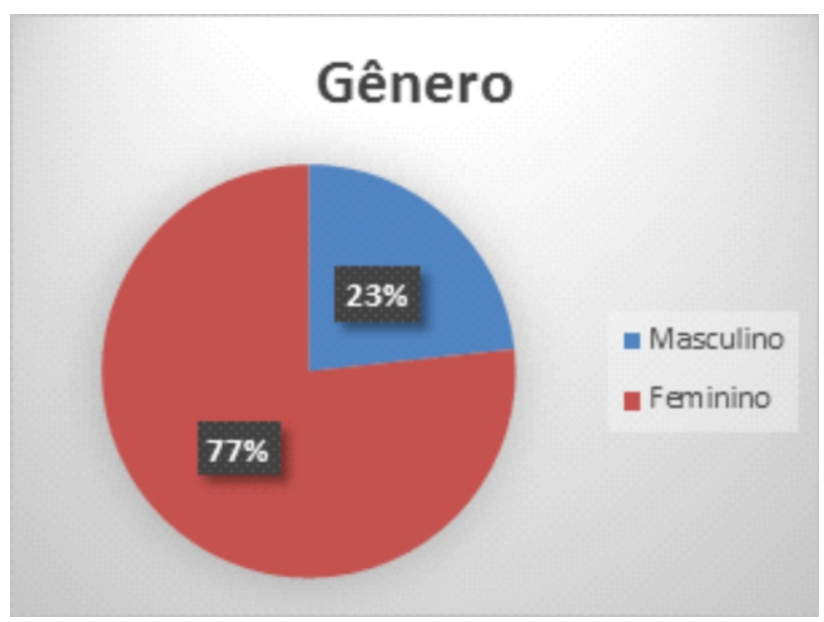

Figura 1 - Distribuição da amostra por gênero. 
Quando questionados sobre a capacidade de atendimento em consultório odontológico no primeiro questionário, cerca de $70 \%$ dos alunos se julgavam capaz de atender as emergências e relataram possuir conhecimento sobre os principais problemas que geram os atendimentos emergenciais em consultório odontológico, entretanto, após a entrega do segundo questionário essa porcentagem foi invertida, embora os alunos tenham conseguido uma taxa de acertos de 70\% (Figura 2).

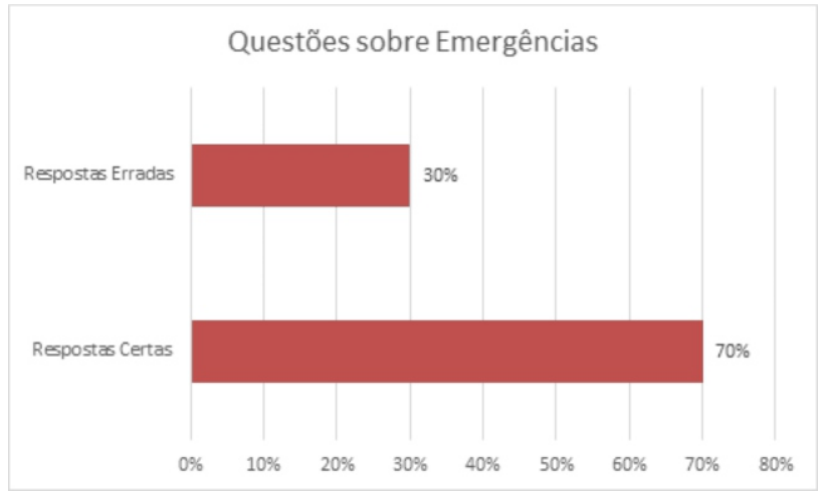

Figura 2 - Porcentagem de erros e acertos das questões avaliativas.

Comparando-se as informações prestadas no primeiro questionário sobre a segurança e capacidade no atendimento das emergências com as respostas do segundo questionário observa-se que após o preenchimento da parte avaliativa os alunos não mantiveram suas respostas, embora tenham conseguido uma porcentagem de acertos considerável (Figura 3).

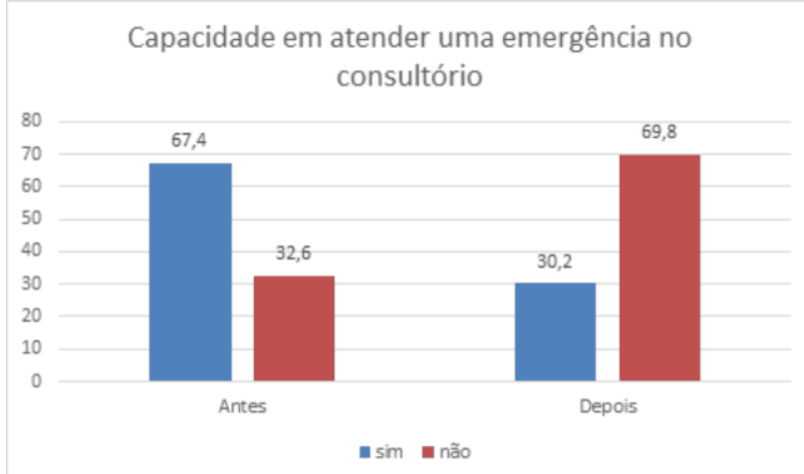

Figura 3 - Comparativo antes e após aplicação do segundo questionário sobre a capacidade em atender uma emergência médica em consultório odontológico.

Após responderem ambos os questionários os $88 \%$ dos acadêmicos se autodeclaram incapazes de atender emergências médicas no consultório levando-se em conta os conhecimentos adquiridos na graduação (Figura 4).

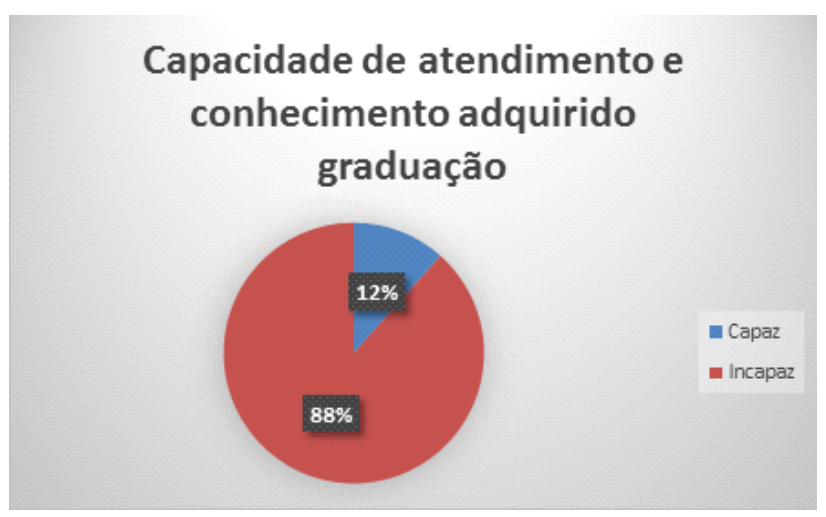

Figura 4 - Capacidade de atendimento de emergências médicas considerando-se o conhecimento adquirido na graduação.

A maioria dos acadêmicos relatou ter interesse em realizar um curso de SBV e que percebem a necessidade de uma disciplina específica abordando a identificação e abordagem de emergências médicas em consultório odontológico (Figura 5).

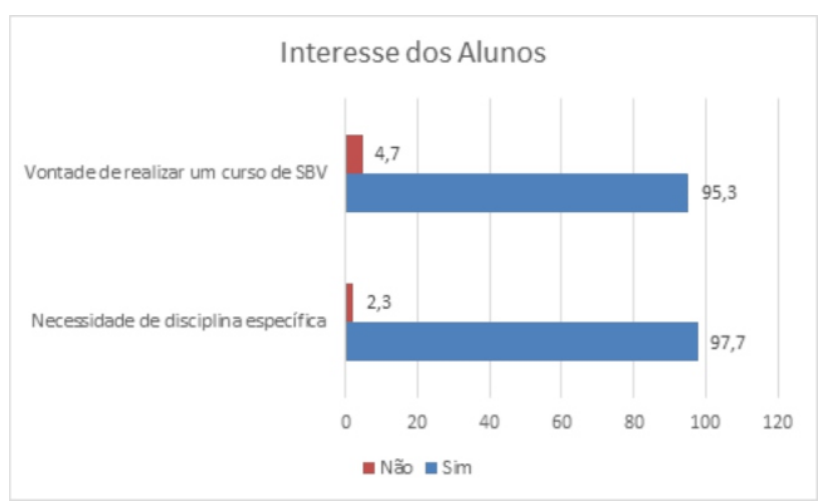

Figura 5 - Interesse dos alunos em realizar curso específico de SBV e necessidade de disciplina específica em SBV.

\section{DISCUSSÃO}

O treinamento constante em SBV deve fazer parte da realidade das faculdades de odontologia de todo o mundo, entretanto no Brasil nem todas as instituições de ensino priorizam o tema primeiros socorros, não abordando 0 assunto de maneira eficiente ${ }^{21}$.

Manobras de ressuscitação e outras medidas em primeiros socorros são abordadas em disciplinas como Anestesiologia, Cirurgia e Traumatologia Bucomaxilofacial, entretanto os alunos acabam não tendo a assimilação adequada das manobras e procedimentos. Embora as técnicas de SBV sejam simples, exige do profissional uma atuação rápida e precisa, pois, como resultado final poderemos ter uma lesão cerebral 
irreversível ou mesmo a morte ${ }^{22-26}$.

O questionário para avaliação do conhecimento dos alunos sobre emergências médicas em consultório odontológico foi interessante para avaliar a assimilação dos alunos sobre o assunto, e ao mesmo tempo evidenciou a insegurança dos alunos, pois, o índice de acertos na parte avaliativa pode ser considerado elevado $70 \%$, entretanto, praticamente $80 \%$ dos alunos mudaram de opinião após o preenchimento do segundo questionário (avaliativo) demostrando dessa forma a insegurança nas respostas e no próprio conhecimento, se diante de um questionário houve esse contrassenso o que poderemos esperar de uma situação real.

As chances de uma emergência médica ocorrer em um consultório odontológico são elevadas, se considerarmos que o cirurgião-dentista realiza procedimentos que promovem estresse, utilizam fármacos, realizam procedimentos cirúrgicos. Qualquer paciente pode ter uma emergência médica a qualquer momento independente do procedimento, incluindo seus acompanhantes, portanto é fundamental que o dentista tenha fundamentação teóricoprática para ter êxito em suas manobras e consequentemente salvar vidas ${ }^{22-26}$.

O ensino continuado e específico se faz necessário, sendo de extrema importância à reciclagem dos conhecimentos e dos protocolos de atendimento com frequência de ao menos dois anos.

\section{CONCLUSÃO}

Os resultados obtidos no presente estudo permitem concluir que os alunos possuem um conhecimento limitado, principalmente teórico sobre as emergências médicas em odontologia, entretanto se faz necessário o treinamento adequado nas manobras em manequins e a reciclagem do conhecimento durante o curso de graduação, promovendo maior conhecimento técnico-cientifico e maior segurança caso seja necessário executar manobras de SBV.

\section{REFERÊNCIAS}

1. Caputo IGC, Bazzo GJ, Silva RHA, Daruge JE. Vidas em risco: emergências médicas em consultório odontológico. Rev Cir Traumatol Buco-maxilo-fac. 2010;10(3):51-8.

2. Brito MAD, Perez FEG, Jorge WA, Borsatti M, Horliana ACRT. Desfalecimento do paciente no consultório odontológico: informações que o cirurgião-dentista deve conhecer. Rev Paul Odontol. 2008;30(1):12-4.

3. Motta RHL, Ramacciato JC, Tófoli GR, Groppo FC. Tratamento odontológico de pacientes usuários de drogas. JBC J Bras Clin Odontol Integr. 2004;8(47):430-8.
4. Queiroga TB, Gomes RC, Novaes MM, Marques JLS, Santos KSA, Grempel RG. Situações de emergências médicas em consultório odontológico. Avaliação das tomada de decisões. Rev Cir Traumatol Buco-maxilo-fac. 2012;12(1):115-22.

5. Gaujac C, Oliveira AN, Barreto FAM, Salgado LM, Oliveira MS, Girão RS. Reações alérgicas medicamentosas no consultório odontológico. Rev Odontol Univ Cidade Sao Paulo. 2009;21(3):268-76.

6. Bordignon MV, Carli BMG, Linden MSS, Silva SO, Carli JP, Trentin MS. Emergências médicas na prática odontológica. Full Dent Sci. 2014;5(18):322-7.

7. Armonia PL, Tortamano N, Ribas TRC, Saraceni JG. Ansiedade e medo - terapêutica medicamentosa. Rev Odontol Univ Santo Amaro. 2001;6:31-4.

8. Possobom RF, Carrascoza KC, Moraes ABA, Costa Jr AL. O tratamento odontológico como gerador de ansiedade. Psicologia Estudo. 2007;12(3):609-16

9. Hanna LMO, Alcântara HSC, Damasceno JM, Santos MTBR. Conhecimento dos cirurgiões-dentistas diante urgência/emergência médica. Rev Cir Traumatol Buco-maxilo-fac. 2001;14(2):79-86.

10. Alhamad M, Alnahwi T, Alshayeb H, Alzayer A, Aldawood O, Almarzouq A, et al. Medical emergencies encountered in dental clinics. A study from the Eastern Province of Saudi Arabia. J Family Community Med. 2015;22(3):175-9.

11. Arsati F, Montalli VA, Flório FM, Ramacciato JC, da Cunha FL, Cecanho R, et al. Brazilian dentists' attitudes about medical emergencies during dental treatment. J Dent Educ. 2010;74(6):661-6.

12. Carvalho RM, Costa LR, Marcelo VC. Brazilian dental students' perceptions about medical emergencies: a qualitative exploratory study. J Dent Educ. 2008;72(11):1343-9.

13. Colet D, Griza GL, Fleig CN, Conci RA, Sinegalia AC. Acadêmicos e profissionais da odontologia estão preparados para salvar vidas? RFO UPF. 2011;16(1): 25-9.

14. Haas DA. Preparing dental office staff members for emergencies developing a basic action plan. J Am Dent Assoc. 2010;141(1):8-13.

15. Lúcio P, Barreto RC. Emergências médicas no consultório odontológico e a (in)segurança dos profissionais. Rev Bras Cienc. Saude. 2012;16(2):267-72.

16. Fiuza MK, Balsan ST, Pretto JLB, Cenci RA, Conto F. Avaliação da prevalência e do grau de conhecimento do cirurgião-dentista em relação às emergências médicas. RFO UPF. 2013;18(3):295-301.

17. Kiffer A, Abreu T. Emergências jurídicas em odontologia. Rev Bras Odontol. 2011;68(1):115-7.

18. Bordignon MV, et al. Emergências médicas na prática odontológica: ocorrência, equipamentos e drogas, conhecimento dos cirurgiõesdentistas do Rio Grande do Sul. Salusvita. 2013;32(2):175-85.

19. Santos JC, Rumel D. Emergência médica na prática odontológica no Estado de Santa Catarina: ocorrência, equipamentos e drogas, conhecimento e treinamento dos cirurgiões-dentistas. Cienc Saude Colet. 2006;11(1):183-90.

20. Ramacciato JC, Motta RHL, Gomes Júnior VQ. Guia de estudo: emergências médicas em odontologia. Campinas: Mundi Brasil; 2007. Jorgensen NB, Hayden JRJ. Sedation, local and general anesthesia in 21. dentistry. 2nd ed. Philadelphia: Lea \& Febiger; 1972. 
22. Victorelli G, et al. Suporte básico de vida e ressuscitação cardiopulmonar em adultos: conceitos atuais e novas recomendações. Rev Assoc Paul Cir Dent. 2013;124-8.

23. Araújo S, Araújo IEM. Ressuscitação cardiorrespiratória. Medicina. 2001;34:36-63.

24. Pazin Filho A, Santos JC, Castro RBP, Bueno CDF, Schmidt A. Parada cardiorrespiratória (PCR). Medicina. 2003;36:163-78.

25. ECC Committee. Subcommittees and Task Forces of the American Heart Association. 2005 American Heart Association guidelines for cardiopulmonary resuscitation and emergency cardiovascular care. Circulation. 2005;112(24 Suppl):IV1-203.

26. Berg RA, Hemphill R, Abella BS, Aufderheide TP, Cave DM, Hazinski MF, et al. Adult basic life support: 2010 American Heart Association guidelines for cardiopulmonary resuscitation and emergency cardiovascular care. Circulation. 2010;(122):685-705. 